\title{
DENJOY-CARLEMAN DIFFERENTIABLE PERTURBATION OF POLYNOMIALS AND UNBOUNDED OPERATORS
}

\author{
ANDREAS KRIEGL, PETER W. MICHOR, AND ARMIN RAINER
}

\begin{abstract}
Let $t \mapsto A(t)$ for $t \in T$ be a $C^{M}$-mapping with values unbounded operators with compact resolvents and common domain of definition which are self-adjoint or normal. Here $C^{M}$ stands for $C^{\omega}$ (real analytic), a quasianalytic or non-quasianalytic Denjoy-Carleman class, $C^{\infty}$, or a Hölder continuity class $C^{0, \alpha}$. The parameter domain $T$ is either $\mathbb{R}$ or $\mathbb{R}^{n}$ or an infinite dimensional convenient vector space. We prove and review results on $C^{M}$-dependence on $t$ of the eigenvalues and eigenvectors of $A(t)$.
\end{abstract}

Theorem. Let $t \mapsto A(t)$ for $t \in T$ be a parameterized family of unbounded operators in a Hilbert space $H$ with common domain of definition and with compact resolvent. If $t \in T=\mathbb{R}$ and all $A(t)$ are self-adjoint then the following holds:

(A) If $A(t)$ is real analytic in $t \in \mathbb{R}$, then the eigenvalues and the eigenvectors of $A(t)$ may be parameterized real analytically in $t$.

(B) If $A(t)$ is quasianalytic of class $C^{Q}$ in $t \in \mathbb{R}$, then the eigenvalues and the eigenvectors of $A(t)$ may be parameterized $C^{Q}$ in $t$.

(C) If $A(t)$ is non-quasianalytic of class $C^{L}$ in $t \in \mathbb{R}$ and if no two unequal continuously parameterized eigenvalues meet of infinite order at any $t \in \mathbb{R}$, then the eigenvalues and the eigenvectors of $A(t)$ can be parameterized $C^{L}$ in $t$.

(D) If $A(t)$ is $C^{\infty}$ in $t \in \mathbb{R}$ and if no two unequal continuously parameterized eigenvalues meet of infinite order at any $t \in \mathbb{R}$, then the eigenvalues and the eigenvectors of $A(t)$ can be parameterized $C^{\infty}$ in $t$.

(E) If $A(t)$ is $C^{\infty}$ in $t \in \mathbb{R}$, then the eigenvalues of $A(t)$ may be parameterized twice differentiably in $t$.

(F) If $A(t)$ is $C^{1, \alpha}$ in $t \in \mathbb{R}$ for some $\alpha>0$, then the eigenvalues of $A(t)$ may be parameterized in a $C^{1}$ way in $t$.

If $t \in T=\mathbb{R}$ and all $A(t)$ are normal then the following holds:

(G) If $A(t)$ is real analytic in $t \in \mathbb{R}$, then for each $t_{0} \in \mathbb{R}$ and for each eigenvalue $\lambda$ of $A\left(t_{0}\right)$ there exists $N \in \mathbb{N}$ such that the eigenvalues near $\lambda$ of $A\left(t_{0} \pm s^{N}\right)$ and their eigenvectors can be parameterized real analytically in $s$ near $s=0$.

$(\mathrm{H})$ If $A(t)$ is $C^{Q}$ in $t \in \mathbb{R}$, then for each $t_{0} \in \mathbb{R}$ and for each eigenvalue $\lambda$ of $A\left(t_{0}\right)$ there exists $N \in \mathbb{N}$ such that the eigenvalues near $\lambda$ of $A\left(t_{0} \pm s^{N}\right)$ and their eigenvectors can be parameterized $C^{Q}$ in $s$ near $s=0$.

(I) If $A(t)$ is $C^{L}$ in $t \in \mathbb{R}$, then for each $t_{0} \in \mathbb{R}$ and for each eigenvalue $\lambda$ of $A\left(t_{0}\right)$ at which no two of the unequal continuously arranged eigenvalues (see [10, II.5.2]) meet of infinite order, there exists $N \in \mathbb{N}$ such that the eigenvalues near $\lambda$ of $A\left(t_{0} \pm s^{N}\right)$ and their eigenvectors can be parameterized $C^{L}$ in $s$ near $s=0$.

Date: November 6, 2018.

2000 Mathematics Subject Classification. 26C10, 26E10, 47A55.

Key words and phrases. Perturbation theory, differentiable choice of eigenvalues and eigenvectors.

PM was supported by FWF-Project P 21030-N13. AR was supported by FWF-Project J2771. 
(J) If $A(t)$ is $C^{\infty}$ in $t \in \mathbb{R}$, then for each $t_{0} \in \mathbb{R}$ and for each eigenvalue $\lambda$ of $A\left(t_{0}\right)$ at which no two of the unequal continuously arranged eigenvalues (see [10. II.5.2]) meet of infinite order, there exists $N \in \mathbb{N}$ such that the eigenvalues near $\lambda$ of $A\left(t_{0} \pm s^{N}\right)$ and their eigenvectors can be parameterized $C^{\infty}$ in $s$ near $s=0$.

(K) If $A(t)$ is $C^{\infty}$ in $t \in \mathbb{R}$ and no two of the unequal continuously parameterized eigenvalues meet of infinite order at any $t \in \mathbb{R}$, then the eigenvalues and the eigenvectors of $A(t)$ can be parameterized by absolutely continuous functions, locally in $t$.

If $t \in T=\mathbb{R}^{n}$ and all $A(t)$ are normal then the following holds:

(L) If $A(t)$ is $C^{\omega}$ or $C^{Q}$ in $t \in \mathbb{R}^{n}$, then for each $t_{0} \in \mathbb{R}^{n}$ and for each eigenvalue $\lambda$ of $A\left(t_{0}\right)$, there exist a finite covering $\left\{\pi_{k}: U_{k} \rightarrow W\right\}$ of a neighborhood $W$ of $t_{0}$, where each $\pi_{k}$ is a composite of finitely many mappings each of which is either a local blow-up along a $C^{\omega}$ or $C^{Q}$ submanifold or a local power substitution, such that the eigenvalues and the eigenvectors of $A\left(\pi_{k}(s)\right)$ can be chosen $C^{\omega}$ or $C^{Q}$ in s. If $A$ is self-adjoint, then we do not need power substitutions.

(M) If $A(t)$ is $C^{\omega}$ or $C^{Q}$ in $t \in \mathbb{R}^{n}$, then the eigenvalues and their eigenvectors of $A(t)$ can be parameterized by functions which are special functions of bounded variation $(S B V)$, see [7] or [2], locally in $t$.

If $t \in T \subseteq E$, a $c^{\infty}$-open subset in an infinite dimensional convenient vector space then the following holds:

(N) For $0<\alpha \leq 1$, if $A(t)$ is $C^{0, \alpha}$ (Hölder continuous of exponent $\alpha$ ) in $t \in T$ and all $A(t)$ are self-adjoint, then the eigenvalues of $A(t)$ may be parameterized in a $C^{0, \alpha}$ way in $t$.

(O) For $0<\alpha \leq 1$, if $A(t)$ is $C^{0, \alpha}$ (Hölder continuous of exponent $\alpha$ ) in $t \in T$ and all $A(t)$ are normal, then we have: For each $t_{0} \in T$ and each eigenvalue $z_{0}$ of $A\left(t_{0}\right)$ consider a simple closed $C^{1}$-curve $\gamma$ in the resolvent set of $A\left(t_{0}\right)$ enclosing only $z_{0}$ among all eigenvalues of $A\left(t_{0}\right)$. Then for $t$ near $t_{0}$ in the $c^{\infty}$-topology on $T$, no eigenvalue of $A(t)$ lies on $\gamma$. Let $\lambda(t)=$ $\left(\lambda_{1}(t), \ldots, \lambda_{N}(t)\right)$ be the $N$-tuple of all eigenvalues (repeated according to their multiplicity) of $A(t)$ inside of $\gamma$. Then $t \mapsto \lambda(t)$ is $C^{0, \alpha}$ for $t$ near $t_{0}$ with respect to the non-separating metric

$$
d(\lambda, \mu)=\min _{\sigma \in \mathcal{S}_{N}} \max _{1 \leq i \leq N}\left|\lambda_{i}-\mu_{\sigma(i)}\right|
$$

on the space of $N$-tuples.

Part (A) is due to Rellich [20] in 1942, see also [3] and [10, VII, 3.9]. Part (D) has been proved in [1, 7.8], see also [11, 50.16], in 1997, which contains also a different proof of $(\mathrm{A})$. (E) and $(\mathrm{F})$ have been proved in $[12$ in 2003. $(\mathrm{G})$ was proved in [18, $7.1]$; it can be proved as $(\mathrm{H})$ with some obvious changes, but it is not a special case since $C^{\omega}$ does not correspond to a sequence which is an $\mathcal{L}$-intersection (see [14]). $(\mathrm{J})$ and $(\mathrm{K})$ were proved in [18, 7.1]. (N) was proved in [15].

The purpose of this paper is to prove the remaining parts $(\mathrm{B}),(\mathrm{C}),(\mathrm{H}),(\mathrm{I}),(\mathrm{L})$, $(\mathrm{M})$, and $(\mathrm{O})$.

Definitions and remarks. Let $M=\left(M_{k}\right)_{k \in \mathbb{N}=\mathbb{N}>0}$ be an increasing sequence $\left(M_{k+1} \geq M_{k}\right)$ of positive real numbers with $M_{0}=1$. Let $U \subseteq \mathbb{R}^{n}$ be open. We denote by $C^{M}(U)$ the set of all $f \in C^{\infty}(U)$ such that, for each compact $K \subseteq U$, there exist positive constants $C$ and $\rho$ such that

$$
\left|\partial^{\alpha} f(x)\right| \leq C \rho^{|\alpha|}|\alpha| ! M_{|\alpha|} \quad \text { for all } \alpha \in \mathbb{N}^{n} \text { and } x \in K \text {. }
$$


The set $C^{M}(U)$ is a Denjoy-Carleman class of functions on $U$. If $M_{k}=1$, for all $k$, then $C^{M}(U)$ coincides with the ring $C^{\omega}(U)$ of real analytic functions on $U$. In general, $C^{\omega}(U) \subseteq C^{M}(U) \subseteq C^{\infty}(U)$.

Here $Q=\left(Q_{k}\right)_{k \in \mathbb{N}}$ is a sequence as above which is quasianalytic, log-convex, and which is also an $\mathcal{L}$-intersection, see 14 or 13 and references therein. Moreover, $L=\left(L_{k}\right)_{k \in \mathbb{N}}$ is a sequence as above which is non-quasianalytic and log-convex.

That $A(t)$ is a real analytic, $C^{M}$ (where $M$ is either $Q$ or $L$ ), $C^{\infty}$, or $C^{k, \alpha}$ family of unbounded operators means the following: There is a dense subspace $V$ of the Hilbert space $H$ such that $V$ is the domain of definition of each $A(t)$, and such that $A(t)^{*}=A(t)$ in the self-adjoint case, or $A(t)$ has closed graph and $A(t) A(t)^{*}=A(t)^{*} A(t)$ wherever defined in the normal case. Moreover, we require that $t \mapsto\langle A(t) u, v\rangle$ is of the respective differentiability class for each $u \in V$ and $v \in H$. ¿From now on we treat only $C^{M}=C^{\omega}, C^{M}$ for $M=Q, M=L$, and $C^{M}=C^{0, \alpha}$.

This implies that $t \mapsto A(t) u$ is of the same class $C^{M}(E, H)$ (where $E$ is either $\mathbb{R}$ or $\mathbb{R}^{n}$ ) or is in $C^{0, \alpha}(E, H)$ (if $E$ is a convenient vector space) for each $u \in V$ by [11, 2.14.4, 10.3] for $C^{\omega}$, by [13, 3.1, 3.3, 3.5] for $M=L$, by [14, $1.10,2.1,2.3$ ] for $M=Q$, and by [11, 2.3], [9, 2.6.2] or [8, 4.14.4] for $C^{0, \alpha}$ because $C^{0, \alpha}$ can be described by boundedness conditions only and for these the uniform boundedness principle is valid.

A sequence of functions $\lambda_{i}$ is said to parameterize the eigenvalues, if for each $z \in \mathbb{C}$ the cardinality $\left|\left\{i: \lambda_{i}(t)=z\right\}\right|$ equals the multiplicity of $z$ as eigenvalue of $A(t)$.

Let $X$ be a $C^{\omega}$ or $C^{Q}$ manifold. A local blow-up $\Phi$ over an open subset $U$ of $X$ means the composition $\Phi=\iota \circ \varphi$ of a blow-up $\varphi: U^{\prime} \rightarrow U$ with center a $C^{\omega}$ or $C^{Q}$ submanifold and of the inclusion $\iota: U \rightarrow X$. A local power substitution is a mapping $\Psi: V \rightarrow X$ of the form $\Psi=\iota \circ \psi$, where $\iota: W \rightarrow X$ is the inclusion of a coordinate chart $W$ of $X$ and $\psi: V \rightarrow W$ is given by

$$
\left(y_{1}, \ldots, y_{q}\right)=\left((-1)^{\epsilon_{1}} x_{1}^{\gamma_{1}}, \ldots,(-1)^{\epsilon_{q}} x_{q}^{\gamma_{q}}\right),
$$

for some $\gamma=\left(\gamma_{1}, \ldots, \gamma_{q}\right) \in\left(\mathbb{N}_{>0}\right)^{q}$ and all $\epsilon=\left(\epsilon_{1}, \ldots, \epsilon_{q}\right) \in\{0,1\}^{q}$, where $y_{1}, \ldots, y_{q}$ denote the coordinates of $W$ (and $\left.q=\operatorname{dim} X\right)$.

This paper became possible only after some of the results of [13] and [14] were proved, in particular the uniform boundedness principles. The wish to prove the results of this paper was the main motivation for us to work on 13 , and 14 .

Applications. Let $X$ be a compact $C^{Q}$ manifold and let $t \mapsto g_{t}$ be a $C^{Q}$-curve of $C^{Q}$ Riemannian metrics on $X$. Then we get the corresponding $C^{Q}$ curve $t \mapsto \Delta\left(g_{t}\right)$ of Laplace-Beltrami operators on $L^{2}(X)$. By theorem (B) the eigenvalues and eigenvectors can be arranged $C^{Q}$. Question: Are the eigenfunctions then also $C^{Q}$ ?

Let $\Omega$ be a bounded region in $\mathbb{R}^{n}$ with $C^{Q}$ boundary, and let $H(t)=-\Delta+V(t)$ be a $C^{Q}$-curve of Schrödinger operators with varying $C^{Q}$ potential and Dirichlet boundary conditions. Then the eigenvalues and eigenvectors can be arranged $C^{Q}$. Question: Are the eigenvectors viewed as eigenfunctions then also in $C^{Q}(\Omega \times \mathbb{R})$ ?

Example. This is an elaboration of [1, 7.4] and [12, Example]. Let $S(2)$ be the vector space of all symmetric real $(2 \times 2)$-matrices. We use the $C^{L}$-curve lemma 13 , 3.6] or [14, 2.5]: There exists a converging sequence of reals $t_{n}$ with the following property: Let $A_{n}, B_{n} \in S(2)$ be any sequences which converge fast to 0, i.e., for each $k \in \mathbb{N}$ the sequences $n^{k} A_{n}$ and $n^{k} B_{n}$ are bounded in $S(2)$. Then there exists a curve $A \in C^{L}(\mathbb{R}, S(2))$ such that $A\left(t_{n}+s\right)=A_{n}+s B_{n}$ for $|s| \leq \frac{1}{n^{2}}$, for all $n$.

We use it for

$$
A_{n}:=\frac{1}{2^{n^{2}}}\left(\begin{array}{cc}
1 & 0 \\
0 & -1
\end{array}\right), \quad B_{n}:=\frac{1}{2^{n^{2}} s_{n}}\left(\begin{array}{ll}
0 & 1 \\
1 & 0
\end{array}\right), \quad \text { where } s_{n}:=2^{n-n^{2}} \leq \frac{1}{n^{2}} .
$$


The eigenvalues of $A_{n}+t B_{n}$ and their derivatives are

$$
\lambda_{n}(t)= \pm \frac{1}{2^{n^{2}}} \sqrt{1+\left(\frac{t}{s_{n}}\right)^{2}}, \quad \lambda_{n}^{\prime}(t)= \pm \frac{2^{n^{2}-2 n} t}{\sqrt{1+\left(\frac{t}{s_{n}}\right)^{2}}} .
$$

Then

$$
\begin{aligned}
\frac{\lambda^{\prime}\left(t_{n}+s_{n}\right)-\lambda^{\prime}\left(t_{n}\right)}{s_{n}^{\alpha}} & =\frac{\lambda_{n}^{\prime}\left(s_{n}\right)-\lambda_{n}^{\prime}(0)}{s_{n}^{\alpha}}= \pm \frac{2^{n^{2}-2 n} s_{n}}{s_{n}^{\alpha} \sqrt{2}} \\
& = \pm \frac{2^{n(\alpha(n-1)-1)}}{\sqrt{2}} \rightarrow \infty \text { for } \alpha>0 .
\end{aligned}
$$

So condition (in $(\mathrm{C}),(\mathrm{D}),(\mathrm{I}),(\mathrm{J})$, and $(\mathrm{K})$ ) that no two unequal continuously parameterized eigenvalues meet of infinite order cannot be dropped. By [1, 2.1], we may always find a twice differentiable square root of a non-negative smooth function, so that the eigenvalues $\lambda$ are functions which are twice differentiable but not $C^{1, \alpha}$ for any $\alpha>0$.

Note that the normed eigenvectors cannot be chosen continuously in this example (see also example [19, §2]). Namely, we have

$$
A\left(t_{n}\right)=A_{n}=\frac{1}{2^{n^{2}}}\left(\begin{array}{cc}
1 & 0 \\
0 & -1
\end{array}\right), \quad A\left(t_{n}+s_{n}\right)=A_{n}+s_{n} B_{n}=\frac{1}{2^{n^{2}}}\left(\begin{array}{cc}
1 & 1 \\
1 & -1
\end{array}\right) .
$$

Resolvent Lemma. Let $C^{M}$ be any of $C^{\omega}, C^{Q}, C^{L}, C^{\infty}$, or $C^{0, \alpha}$, and let $A(t)$ be normal. If $A$ is $C^{M}$ then the resolvent $(t, z) \mapsto(A(t)-z)^{-1} \in L(H, H)$ is $C^{M}$ on its natural domain, the global resolvent set

$$
\{(t, z) \in T \times \mathbb{C}:(A(t)-z): V \rightarrow H \text { is invertible }\}
$$

which is open (and even connected).

Proof. By definition the function $t \mapsto\langle A(t) v, u\rangle$ is of class $C^{M}$ for each $v \in V$ and $u \in H$. We may conclude that the mapping $t \mapsto A(t) v$ is of class $C^{M}$ into $H$ as follows: For $C^{M}=C^{\infty}$ we use [11, 2.14.4]. For $C^{M}=C^{\omega}$ we use in addition [11, 10.3]. For $C^{M}=C^{Q}$ or $C^{M}=C^{L}$ we use [14, 2.1] and/or [13, 3.3] where we replace $\mathbb{R}$ by $\mathbb{R}^{n}$. For $C^{M}=C^{0, \alpha}$ we use [11, 2.3], [9, 2.6.2], or [8, 4.1.14] because $C^{0, \alpha}$ can be described by boundedness conditions only and for these the uniform boundedness principle is valid.

For each $t$ consider the norm $\|u\|_{t}^{2}:=\|u\|^{2}+\|A(t) u\|^{2}$ on $V$. Since $A(t)$ is closed, $\left(V,\|\|_{t}\right)$ is again a Hilbert space with inner product $\langle u, v\rangle_{t}:=\langle u, v\rangle+$ $\langle A(t) u, A(t) v\rangle$.

(1) Claim (see [1, in the proof of 7.8], 11, in the proof of 50.16], or 12, Claim 1]). All these norms \|\|$_{t}$ on $V$ are equivalent, locally uniformly in $t$. We then equip $V$ with one of the equivalent Hilbert norms, say \|\|$_{0}$.

We reduce this to $C^{0, \alpha}$. Namely, note first that $A(t):\left(V,\|\|_{s}\right) \rightarrow H$ is bounded since the graph of $A(t)$ is closed in $H \times H$, contained in $V \times H$ and thus also closed in $\left(V,\|\|_{s}\right) \times H$. For fixed $u, v \in V$, the function $t \mapsto\langle u, v\rangle_{t}=\langle u, v\rangle+$ $\langle A(t) u, A(t) v\rangle$ is $C^{0, \alpha}$ since $t \mapsto A(t) u$ is it. By the multilinear uniform boundedness principle $\left([11,5.18]\right.$ or [9, 3.7.4]) the mapping $t \mapsto\langle, \quad\rangle_{t}$ is $C^{0, \alpha}$ into the space of bounded sesquilinear forms on $\left(V,\|\|_{s}\right)$ for each fixed $s$. Thus the inverse image of $\langle, \quad\rangle_{s}+\frac{1}{2}$ (unit ball) in $L\left(\overline{\left(V,\|\|_{s}\right)} \oplus\left(V,\|\|_{s}\right) ; \mathbb{C}\right)$ is a $c^{\infty}$-open neighborhood $U$ of $s$ in $T$. Thus $\sqrt{1 / 2}\|u\|_{s} \leq\|u\|_{t} \leq \sqrt{3 / 2}\|u\|_{s}$ for all $t \in U$, i.e. all Hilbert norms $\|\quad\|_{t}$ are locally uniformly equivalent, and claim (1) follows.

By the linear uniform boundedness theorem we see that $t \mapsto A(t)$ is in $C^{M}(T, L(V, H))$ as follows (here it suffices to use a set of linear functionals which together recognize bounded sets instead of the whole dual): For $C^{M}=C^{\infty}$ we use 
11, 1.7 and 2.14.3]. For $C^{M}=C^{\omega}$ we use in addition [11, 9.4]. For $C^{M}=C^{Q}$ or $C^{M}=C^{L}$ we use [14, 2.2 and 2.3] and/or [13, 3.5] where we replace $\mathbb{R}$ by $\mathbb{R}^{n}$. For $C^{M}=C^{0, \alpha}$ see above.

If for some $(t, z) \in T \times \mathbb{C}$ the bounded operator $A(t)-z: V \rightarrow H$ is invertible, then this is true locally with respect to the $c^{\infty}$-topology on the product which is the product topology by [11, 4.16], and $(t, z) \mapsto(A(t)-z)^{-1}: H \rightarrow V$ is $C^{M}$, by the chain rule, since inversion is real analytic on the Banach space $L(V, H)$.

Note that $(A(t)-z)^{-1}: H \rightarrow H$ is a compact operator for some (equivalently any) $(t, z)$ if and only if the inclusion $i: V \rightarrow H$ is compact, since $i=(A(t)-z)^{-1} \circ$ $(A(t)-z): V \rightarrow H \rightarrow H$.

Polynomial proposition. Let $P$ be a curve of polynomials

$$
P(t)(x)=x^{n}-a_{1}(t) x^{n-1}+\cdots+(-1)^{n} a_{n}(t), \quad t \in \mathbb{R} .
$$

(a) If $P$ is hyperbolic (all roots real) and if the coefficient functions $a_{i}$ are all $C^{Q}$ then there exist $C^{Q}$ functions $\lambda_{i}$ which parameterize all roots.

(b) If $P$ is hyperbolic (all roots real), if the coefficient functions $a_{i}$ are $C^{L}$ and no two of the different roots meet of infinite order, then there exist $C^{L}$ functions $\lambda_{i}$ which parameterize all roots.

(c) If the coefficient functions $a_{i}$ are $C^{Q}$, then for each $t_{0}$ there exists $N \in \mathbb{N}$ such that the roots of $s \mapsto P\left(t_{0} \pm s^{N}\right)$ can be parameterized $C^{Q}$ in $s$ for $s$ near 0.

(d) If the coefficient functions $a_{i}$ are $C^{L}$ and no two of the different roots meet of infinite order, then for each $t_{0}$ there exists $N \in \mathbb{N}$ such that the roots of $s \mapsto P\left(t_{0} \pm s^{N}\right)$ can be parameterized $C^{L}$ in $s$ for $s$ near 0.

All $C^{Q}$ or $C^{L}$ solutions differ by permutations.

The proof of parts (a) and (b) is exactly as in [1] where the corresponding results were proven for $C^{\infty}$ instead of $C^{L}$, and for $C^{\omega}$ instead of $C^{Q}$. For this we need only the following properties of $C^{Q}$ and $C^{L}$ :

- They allow for the implicit function theorem (for [1, 3.3]).

- They contain $C^{\omega}$ and are closed under composition (for [1, 3.4]).

- They are derivation closed (for [1, 3.7]).

Part (a) is also in [6, 7.6] which follows [1. It also follows from the multidimensional version [17, 6.10] since blow-ups in dimension 1 are trivial. The proofs of parts (c) and (d) are exactly as in [18, 3.2] where the corresponding result was proven for $C^{\omega}$ instead of $C^{Q}$, and for $C^{\infty}$ instead of $C^{L}$, if none of the different roots meet of infinite order. For these we need the properties of $C^{Q}$ and $C^{L}$ listed above.

Matrix proposition. Let $A(t)$ for $t \in T$ be a family of $(N \times N)$-matrices.

(e) If $T=\mathbb{R} \ni t \mapsto A(t)$ is a $C^{Q}$-curve of Hermitian matrices, then the eigenvalues and the eigenvectors can be chosen $C^{Q}$.

(f) If $T=\mathbb{R} \ni t \mapsto A(t)$ is a $C^{L}$-curve of Hermitian matrices such that no two eigenvalues meet of infinite order, then the eigenvalues and the eigenvectors can be chosen $C^{L}$.

(g) If $T=\mathbb{R} \ni t \mapsto A(t)$ is a $C^{L}$-curve of normal matrices such that no two eigenvalues meet of infinite order, then for each $t_{0}$ there exists $N_{1} \in$ $\mathbb{N}$ such that the eigenvalues and eigenvectors of $s \mapsto A\left(t_{0} \pm s^{N_{1}}\right)$ can be parameterized $C^{L}$ in $s$ for $s$ near 0 .

(h) Let $T \subseteq \mathbb{R}^{n}$ be open and let $T \ni t \mapsto A(t)$ be a $C^{\omega}$ or $C^{Q}$-mapping of normal matrices. Let $K \subseteq T$ be compact. Then there exist a neighborhood $W$ of $K$, and a finite covering $\left\{\pi_{k}: U_{k} \rightarrow W\right\}$ of $W$, where each $\pi_{k}$ is a composite of finitely many mappings each of which is either a local blowup along a $C^{\omega}$ or $C^{Q}$ submanifold or a local power substitution, such that 
the eigenvalues and the eigenvectors of $A\left(\pi_{k}(s)\right)$ can be chosen $C^{\omega}$ or $C^{Q}$ in s. Consequently, the eigenvalues and eigenvectors of $A(t)$ are locally special functions of bounded variation (SBV). If $A$ is a family of Hermitian matrices, then we do not need power substitutions.

The proof of the matrix proposition in case (e) and (f) is exactly as in [1, 7.6], using the polynomial proposition and properties of $C^{Q}$ and $C^{L}$. Item (g) is exactly as in [18, 6.2], using the polynomial proposition and properties of $C^{L}$. Item (h) is proved in [17, 9.1 and 9.6], see also [16].

Proof of the theorem. We have to prove parts (B), (C), (H), (I), (L), (M), and (O). So let $C^{M}$ be any of $C^{\omega}, C^{Q}, C^{L}$, or $C^{0, \alpha}$, and let $A(t)$ be normal. Let $z$ be an eigenvalue of $A\left(t_{0}\right)$ of multiplicity $N$. We choose a simple closed $C^{1}$ curve $\gamma$ in the resolvent set of $A\left(t_{0}\right)$ for fixed $t_{0}$ enclosing only $z$ among all eigenvalues of $A\left(t_{0}\right)$. Since the global resolvent set is open, see the resolvent lemma, no eigenvalue of $A(t)$ lies on $\gamma$, for $t$ near $t_{0}$. By the resolvent lemma, $A: T \rightarrow L\left(\left(V,\|\|_{0}\right), H\right)$ is $C^{M}$, thus also

$$
t \mapsto-\frac{1}{2 \pi i} \int_{\gamma}(A(t)-z)^{-1} d z=: P(t, \gamma)=P(t)
$$

is a $C^{M}$ mapping. Each $P(t)$ is a projection, namely onto the direct sum of all eigenspaces corresponding to eigenvalues of $A(t)$ in the interior of $\gamma$, with finite rank. Thus the rank must be constant: It is easy to see that the (finite) rank cannot fall locally, and it cannot increase, since the distance in $L(H, H)$ of $P(t)$ to the subset of operators of rank $\leq N=\operatorname{rank}\left(P\left(t_{0}\right)\right)$ is continuous in $t$ and is either 0 or 1 .

So for $t$ in a neighborhood $U$ of $t_{0}$ there are equally many eigenvalues in the interior of $\gamma$, and we may call them $\lambda_{i}(t)$ for $1 \leq i \leq N$ (repeated with multiplicity).

Now we consider the family of $N$-dimensional complex vector spaces $t \mapsto$ $P(t)(H) \subseteq H$, for $t \in U$. They form a $C^{M}$ Hermitian vector subbundle over $U$ of $U \times H \rightarrow U$ : For given $t$, choose $v_{1}, \ldots v_{N} \in H$ such that the $P(t) v_{i}$ are linearly independent and thus span $P(t) H$. This remains true locally in $t$. Now we use the Gram Schmidt orthonormalization procedure (which is $C^{\omega}$ ) for the $P(t) v_{i}$ to obtain a local orthonormal $C^{M}$ frame of the bundle.

Now $A(t)$ maps $P(t) H$ to itself; in a $C^{M}$ local frame it is given by a normal $(N \times N)$-matrix parameterized $C^{M}$ by $t \in U$.

Now all local assertions of the theorem follow:

(B) Use the matrix proposition, part (e).

(C) Use the matrix proposition, part (f).

(H) Use the matrix proposition, part (h), and note that in dimension 1 blowups are trivial.

(I) Use the matrix proposition, part (g).

$(\mathrm{L}, \mathrm{M})$ Use the matrix proposition, part $(\mathrm{h})$, for $\mathbb{R}^{n}$.

(O) We use the following

Result ([5], 4, VII.4.1]) Let $A, B$ be normal $(N \times N)$-matrices and let $\lambda_{i}(A)$ and $\lambda_{i}(B)$ for $i=1, \ldots, N$ denote the respective eigenvalues. Then

$$
\min _{\sigma \in \mathcal{S}_{N}} \max _{j}\left|\lambda_{j}(A)-\lambda_{\sigma(j)}(B)\right| \leq C\|A-B\|
$$

for a universal constant $C$ with $1<C<3$. Here \|\| is the operator norm.

Finally, it remains to extend the local choices to global ones for the cases (B) and (C) only. There $t \mapsto A(t)$ is $C^{Q}$ or $C^{L}$, respectively, which imply both $C^{\infty}$, and no two different eigenvalues meet of infinite order. So we may apply [1, 7.8] (in fact we need only the end of the proof) to conclude that the eigenvalues can be chosen $C^{\infty}$ on $T=\mathbb{R}$, uniquely up to a global permutation. By the local result 
above they are then $C^{Q}$ or $C^{L}$. The same proof then gives us, for each eigenvalue $\lambda_{i}: T \rightarrow \mathbb{R}$ with generic multiplicity $N$, a unique $N$-dimensional smooth vector subbundle of $\mathbb{R} \times H$ whose fiber over $t$ consists of eigenvectors for the eigenvalue $\lambda_{i}(t)$. In fact this vector bundle is $C^{Q}$ or $C^{L}$ by the local result above, namely the matrix proposition, part (e) or (f), respectively.

\section{REFERENCES}

[1] D. Alekseevsky, A. Kriegl, M. Losik, and P. W. Michor, Choosing roots of polynomials smoothly, Israel J. Math. 105 (1998), 203-233. MR 1639759 (2000c:58017)

[2] L. Ambrosio, N. Fusco, and D. Pallara, Functions of bounded variation and free discontinuity problems, Oxford Mathematical Monographs, The Clarendon Press Oxford University Press, New York, 2000. MR 1857292 (2003a:49002)

[3] H. Baumgärtel, Endlichdimensionale analytische Störungstheorie, Akademie-Verlag, Berlin, 1972, Mathematische Lehrbücher und Monographien, II. Abteilung. Mathematische Monographien, Band 28. MR 0634965 (58 \#30389)

[4] R. Bhatia, Matrix analysis, Graduate Texts in Mathematics, vol. 169, Springer-Verlag, New York, 1997. MR 1477662 (98i:15003)

[5] R. Bhatia, C. Davis, and A. McIntosh, Perturbation of spectral subspaces and solution of linear operator equations, Linear Algebra Appl. 52/53 (1983), 45-67. MR 709344 (85a:47020)

[6] J. Chaumat and A.-M. Chollet, Division par un polynôme hyperbolique, Canad. J. Math. 56 (2004), no. 6, 1121-1144. MR 2102628 (2005g:32008)

[7] E. De Giorgi and L. Ambrosio, New functionals in the calculus of variations, Atti Accad. Naz. Lincei Rend. Cl. Sci. Fis. Mat. Natur. (8) 82 (1988), no. 2, 199-210 (1989). MR 1152641 (92j:49043)

[8] C.-A. Faure, Théorie de la différentiation dans les espaces convenables, Ph.D. thesis, Université de Genéve, 1991.

[9] A. Frölicher and A. Kriegl, Linear spaces and differentiation theory, Pure and Applied Mathematics (New York), John Wiley \& Sons Ltd., Chichester, 1988, A Wiley-Interscience Publication. MR 961256 (90h:46076)

[10] T. Kato, Perturbation theory for linear operators, second ed., Grundlehren der Mathematischen Wissenschaften, vol. 132, Springer-Verlag, Berlin, 1976. MR 0407617 (53 \#11389)

[11] A. Kriegl and P. W. Michor, The convenient setting of global analysis, Mathematical Surveys and Monographs, vol. 53, American Mathematical Society, Providence, RI, 1997, http://www.ams.org/online_bks/surv53/. MR 1471480 (98i:58015)

[12] Differentiable perturbation of unbounded operators, Math. Ann. 327 (2003), no. 1, 191-201. MR 2006008 (2005e:47030)

[13] A. Kriegl, P. W. Michor, and A. Rainer, The convenient setting for non-quasianalytic Denjoy-Carleman differentiable mappings, J. Funct. Anal. 256 (2009), 3510-3544.

[14] The convenient setting for quasianalytic Denjoy-Carleman differentiable mappings, Preprint, arXiv:0909.5632v1, 2009.

[15] - Many parameter Hölder perturbation of unbounded operators, Preprint, arXiv:math.FA/0611506, 2009.

[16] K. Kurdyka and L. Paunescu, Hyperbolic polynomials and multiparameter real-analytic perturbation theory, Duke Math. J. 141 (2008), no. 1, 123-149. MR 2372149 (2009d:32003)

[17] A. Rainer, Quasianalytic multiparameter perturbation of polynomials and normal matrices, Preprint, arXiv:0905.0837, 2009.

[18] Perturbation of complex polynomials and normal operators, to appear in Math. Nach. 283 (2010), arXiv:math.CA/0611633, 2010.

[19] F. Rellich, Störungstheorie der Spektralzerlegung, Math. Ann. 113 (1937), no. 1, 600-619. MR 1513109

[20] _ Störungstheorie der Spektralzerlegung. V, Math. Ann. 118 (1942), 462-484. MR $0010791(6,71 \mathrm{e})$

Andreas Kriegl: Fakultät für Mathematik, Universität Wien, Nordbergstrasse 15, A-1090 Wien, Austria

E-mail address: andreas.kriegl@univie.ac.at

Peter W. Michor: Fakultät für Mathematik, Universität Wien, Nordbergstrasse 15, A-1090 Wien, Austria

E-mail address: peter.michor@univie.ac.at 
Armin Rainer: Department of Mathematics, University of Toronto, 40 St. George Street, Toronto, Ontario, Canada M5S 2E4

E-mail address: armin.rainer@univie.ac.at 